\title{
Philippe Collas (ed): Chromatin immunoprecipitation assays: methods and protocols
}

\author{
Springer, 2009, HB, 267 pages, Price: \$99.00. ISBN: 978-1-60327-413-5
}

\author{
Ray Waters
}

Published online: 9 March 2010

(C) Springer-Verlag 2010

This is a very useful book for researchers who utilise chromatin Immunoprecipitation (ChIP). Not only does it describe a range of applications and methods in detail, but it also considers a number of hurdles encountered by the researchers employing ChIP and it describes how many of these can be overcome. The focus is on events in mammalian cells with some description of research with zebrafish.

The only criticism I have is that ChIP has been extensively employed in other model organisms, and as a yeast geneticist, I would have liked chapters on the use of ChIP in these organisms!

The opening chapter is very well written and it introduces the reader to ChIP; it has an extensive bibliography. The second chapter considers the characterisation and quality control of antibodies for ChIP. This is an important topic and it is reassuring to see detailed protocols to undertake such QC.

The third chapter considers a fast ChIP approach that would be of use to some researchers. This is followed by a chapter on $\mu \mathrm{ChIP}$; an approach for chromatin immunoprecipitation with cell numbers ranging from 100 to 100,000. Next comes a chapter on undertaking ChIP in the zebrafish embryo, and this is followed by a chapter that concerns the epitope tagging of endogenous proteins for genome-wide ChIP analysis with human cells. Hence this chapter provides a strategy to examine proteins for which antibodies are unavailable or are sub-standard. Given that this is not an uncommon occurrence when undertaking ChIP, this is a section that will be useful to many

R. Waters $(\square)$

Pathology Department, Cardiff University, Tenovus Building,

Heath Park, Cardiff, UK

e-mail: watersr1@Cardiff.ac.uk researchers working with mammalian cells. It would have been good to see cross reference to a range of tagging approaches that can be employed in specific organisms, but bibliography is woefully short and no such references are cited.

Chapter 7 considers flow cytometric and laser scanning approaches for researching epigenetics, and this is followed by a chapter considering the serial analysis of the binding elements for transcription factors. Chapter 9 concerns a central facet of ChIP-chip experiments, namely the modelling and analysis of data. The computational aspects of using SNP-arrays for ChIP assays are then discussed.

Employing Dam ID to examine genomic binding sites is then covered. This is undertaken by fusing a bacterial Dam-methylase to the protein of interest and then identifying its genomic binding sites via adenine methylation. Then we have two chapters on Chromosome Conformation Capture (3C); the first introduces $3 \mathrm{C}$, and its $4 \mathrm{C}$ and $5 \mathrm{C}$ adaptations, along with its ChIP-based modification. The second of these chapters concerns determining the spatial chromatin organization of large genomic regions using $5 \mathrm{C}$ technology.

The book then goes on to cover the analysis of nascent RNA transcripts via chromatin RNA IP. The last two chapters discuss methyl DNA immunoprecipitation and the immunoprecipitation of methylated DNA.

In conclusion, the chapters in this book describe the methods in detail and in general they have reasonable bibliographies. This book is a very useful acquisition for researchers who are embarking upon research that employs ChIP and equally for those researchers who are already undertaking it, but who may wish to expand the applications which they currently employ. 